\title{
THE SPACE-LIKE SURFACES WITH VANISHING CONFORMAL FORM IN THE CONFORMAL SPACE
}

\author{
CHANGXIONG NIE
}

\begin{abstract}
The conformal geometry of surfaces in the conformal space $\mathbf{Q}_{1}^{n}$ is studied. We classify the space-like surfaces in $\mathbf{Q}_{1}^{n}$ with vanishing conformal form up to conformal equivalence.
\end{abstract}

\section{INTRODUCTION}

In [1] Wang gave the structure equations for Möbius geometry of submanifolds in the unit sphere. Three fundamental tensors $\mathbb{A}, \mathbb{B}$ and $\Phi$ arise naturally in the structure equations. In $[\mathbb{A}$ is called the Blaschke tensor, $\mathbb{B}$ the Möbius second fundamental form, and $\Phi$ the Möbius form. Together with Möbius metric $g$, these tensors determine the submanifold up to Möbius transformations of the unit sphere.

$\mathrm{Li}$ and Wang [2] classified surfaces with vanishing Möbius form in sphere space $\mathbb{S}^{n+1}$. Readers should be reminded that Bryant [3] have classified all minimal surfaces with constant curvature in the unit sphere $\mathbf{S}^{n}$, the hyperbolic space $\mathbf{H}^{n}$ and Euclidean space $\mathbf{R}^{n}$. Li and Wang used Bryant's results. It is interesting to classify stationary surfaces with constant curvature in $\mathbf{R}_{1}^{n}, \mathbf{S}_{1}^{n}$ or $\mathbf{H}_{1}^{n}$. For some other results about Lorentz conformal geometry, see [4]-7]. Further relative knowledge refers to [8]-10].

We conclude this paper with the following.

The Main Theorem. Let $x: \mathbf{M} \rightarrow \mathbf{Q}_{1}^{n}$ be a regular spacelike full surface with vanishing form. Then $x$ is one of the following four alternatives:

(i) $x$ is a stationary surface with constant curvature in $\mathbf{R}_{1}^{n}, \mathbf{S}_{1}^{n}$ or $\mathbf{H}_{1}^{n}$.

(ii) $x$ is a hyperbolic cylinder $\mathbf{H}^{1} \times \mathbf{R}$ in $\mathbf{R}_{1}^{3}$.

(iii) $x$ is a surface $\mathbf{H}^{1}(\sqrt{r}) \times \mathbf{S}^{1}(\sqrt{1+r})$ in $\mathbf{S}_{1}^{3}, r>0$.

(iv) $x$ is a hyperbolic torus $\mathbf{H}^{1}(\sqrt{1+r}) \times \mathbf{H}^{1}(\sqrt{-r})$ in $\mathbf{H}_{1}^{3},-\frac{1}{2} \leq r<0$.

\section{The FUndamental EQUATIONS}

Let $\mathbb{R}_{s}^{n}$ be the real vector space $\mathbb{R}^{n}$ with the Lorentzian inner product $\langle$,$\rangle given$ by

$$
\langle X, Y\rangle:=\sum_{i=1}^{n-s} x_{i} y_{i}-\sum_{i=n-s+1}^{n} x_{i} y_{i}
$$

Received by the editors July 29, 2011.

2010 Mathematics Subject Classification. Primary 53A30, 53C50.

This work was partially supported by National Natural Science Foundation of China (Grant Nos. 10971055 and 10801006) and Zhongdian Natural Science Foundation of Hubei Educational Committee. 
where $X=\left(x_{1}, \cdots x_{n}\right), Y=\left(y_{1}, \cdots, y_{n}\right) \in \mathbb{R}^{n}$. We define the de Sitter sphere $\mathbf{S}_{1}^{n}$ and anti-de Sitter sphere $\mathbf{H}_{1}^{n}$ by

$$
\mathbf{S}_{1}^{n}=\left\{u \in \mathbf{R}_{1}^{n+1} \mid\langle u, u\rangle=1\right\}, \quad \mathbf{H}_{1}^{n}=\left\{u \in \mathbf{R}_{2}^{n+1} \mid\langle u, u\rangle=-1\right\} .
$$

We call Lorentzian space $\mathbf{R}_{1}^{n}$, de Sitter sphere $\mathbf{S}_{1}^{n}$ and anti-de Sitter sphere $\mathbf{H}_{1}^{n}$ Lorentzian space forms. Denote

$$
\mathbf{Q}_{1}^{n}=\left\{[x] \text { is the projective coordinates } \mid\langle x, x\rangle=0, x \in \mathbf{R}_{2}^{n+2}\right\} \text {. }
$$

By some conformal diffeomorphisms we may regard $\mathbf{Q}_{1}^{n}$ as the common compactified space of $\mathbf{R}_{1}^{n}, \mathbf{S}_{1}^{n}$ and $\mathbf{H}_{1}^{n}$. In fact the conformal space $\mathbf{Q}_{1}^{n}$ has a standard Lorentzian metric. We research the conformal geometry of surfaces under the conformal group of this Lorentzian metric. We refer the reader to [4] and [7] for further details.

Suppose that $x: \mathbf{M} \rightarrow \mathbf{Q}_{1}^{n}$ is a space-like surface. That is, $x_{*}(\mathrm{TM})$ is a nondegenerated subbundle of $\mathbf{T} \mathbf{Q}_{1}^{n}$. Let $y: U \rightarrow \mathbf{R}_{2}^{n+2}$ be a lift of $x: \mathbf{M} \rightarrow \mathbf{Q}_{1}^{n}$ defined in an open subset $U$ of $\mathbf{M}$. We denote by $\Delta$ and $\kappa$, Laplacian and the normalized scalar curvature of the local positive definite metric $\langle d y, d y\rangle$. Then we have the following theorem.

Theorem 2.1 (see [1, Theorem 1.2). On $\mathbf{M}$ the 2-form $g=-(\langle\Delta y, \Delta y\rangle-$ $4 \kappa)\langle d y, d y\rangle$ is a globally defined invariant of $x: \mathbf{M} \rightarrow \mathbf{Q}_{1}^{n}$ under the Lorentz group transformations of $\mathbf{Q}_{1}^{n}$.

Let $x: \mathbf{M}^{2} \rightarrow \mathbf{Q}_{1}^{n}$ be a regular space-like surface. That is, the 2-form $g=$ $-(\langle\Delta y, \Delta y\rangle-4 \kappa)\langle d y, d y\rangle$, which is called a conformal metric, is non-degenerated. Let $Y=\sqrt{-(\langle\Delta y, \Delta y\rangle-4 \kappa)} y$ be the canonical lift of $x$ and define $N: \mathbf{M} \rightarrow \mathbf{R}_{2}^{n+2}$ by $N=-\frac{1}{2} \Delta Y-\frac{1}{8}\langle\Delta Y, \Delta Y\rangle Y$. Let $\left\{E_{\alpha}\right\}$ be a local basis of the conformal normal bundle $\mathbf{V}$ of $x$. If $z=u+\mathrm{i} v$ is a local isothermal coordinate on $\mathbf{M}$ for $g$, we can write $g=e^{2 \omega}|d z|^{2}=\frac{1}{2} e^{2 \omega}(d z \otimes d \bar{z}+d \bar{z} \otimes d z)$ for some local smooth function $\omega$. Denote by $K$ the Gauss curvature of $g$; we have $\Delta Y=4 e^{-2 \omega} Y_{z \bar{z}}, K=-4 e^{-2 \omega} \omega_{z \bar{z}}$. Since $\left\{Y, N, \operatorname{Re}\left(Y_{z}\right), \operatorname{Im}\left(Y_{z}\right), E_{\alpha}\right\}$ is a moving frame in $\mathbf{R}_{2}^{n+2}$ along $\mathbf{M}$, one can write the structure equations and the fundamental equations as in [2]. Define

$$
\psi=2\left\langle N_{z}, Y_{z}\right\rangle, \quad \phi_{\alpha}=\left\langle N_{z}, E_{\alpha}\right\rangle, \quad \Omega_{\alpha}=2\left\langle Y_{z z}, E_{\alpha}\right\rangle, \quad A_{\alpha \beta}=\left\langle\left(E_{\alpha}\right)_{z}, E_{\beta}\right\rangle
$$

and $\phi^{\alpha}=\sum_{\beta} g^{\alpha \beta} \phi_{\beta}, \quad \Omega^{\alpha}=\sum_{\beta} g^{\alpha \beta} \Omega_{\beta}, \quad A_{\alpha}^{\beta}=\sum_{\gamma} g^{\beta \gamma} A_{\alpha \gamma}$. The structure equations are

$$
\begin{aligned}
N_{z} & =\frac{1}{8}(4 K-1) Y_{z}+e^{-2 \omega} \psi Y_{\bar{z}}+\sum_{\alpha} \phi^{\alpha} E_{\alpha}, \\
Y_{z z} & =-\frac{1}{2} \psi Y+2 \omega_{z} Y_{z}+\frac{1}{2} \sum_{\alpha} \Omega^{\alpha} E_{\alpha} \\
Y_{z \bar{z}} & =-\frac{1}{16} e^{2 \omega}(4 K-1) Y-\frac{1}{2} e^{2 \omega} N \\
\left(E_{\alpha}\right)_{z} & =-\phi_{\alpha} Y-e^{-2 \omega} \Omega_{\alpha} Y_{\bar{z}}+\sum_{\beta} A_{\alpha}^{\beta} E_{\beta} .
\end{aligned}
$$


The fundamental equations are

$$
\begin{aligned}
\psi_{\bar{z}} & =\frac{1}{2} e^{2 \omega} K_{z}-\sum_{\alpha} \Omega^{\alpha} \bar{\phi}_{\alpha}, \sum_{\alpha} \Omega^{\alpha} \Omega_{\alpha} \\
& =-\frac{1}{4} e^{4 \omega},\left(\Omega_{\alpha}\right)_{\bar{z}}=-\sum_{\beta} \Omega^{\beta} \bar{A}_{\beta \alpha}-e^{2 \omega} \phi_{\alpha} \\
\left(\phi_{\alpha}\right)_{\bar{z}} & -\frac{1}{2} e^{-2 \omega} \bar{\psi} \Omega_{\alpha}+\sum_{\beta} \phi^{\beta} \bar{A}_{\beta \alpha}=\left(\bar{\phi}_{\alpha}\right)_{z}-\frac{1}{2} e^{-2 \omega} \psi \bar{\Omega}_{\alpha}+\sum_{\beta} \bar{\phi}^{\beta} A_{\beta \alpha} \\
\left(A_{\alpha \beta}\right)_{\bar{z}} & -\left(\bar{A}_{\alpha \beta}\right)_{z}=\frac{1}{2} e^{-2 \omega}\left(\Omega_{\alpha} \bar{\Omega}_{\beta}-\bar{\Omega}_{\alpha} \Omega_{\beta}\right)+\sum_{\gamma}\left(\bar{A}_{\alpha \gamma} A_{\beta}^{\gamma}-A_{\alpha \gamma} \bar{A}_{\beta}^{\gamma}\right)
\end{aligned}
$$

Remark 2.1. $\Psi=\psi d z \otimes d z, \Phi=\sum_{\alpha}\left(\phi^{\alpha} d z+\bar{\phi}^{\alpha} d \bar{z}\right) \otimes E_{\alpha}$ and $\Omega=\sum_{\alpha} \Omega^{\alpha} d z \otimes d z \otimes E_{\alpha}$ are globally defined conformal invariants.

Remark 2.2. The Willmore equations are

$$
\left(\phi_{\alpha}\right)_{\bar{z}}-\frac{1}{2} e^{-2 \omega} \bar{\psi} \Omega_{\alpha}+\sum_{\beta} \phi^{\beta} \bar{A}_{\beta \alpha}=0, \forall \alpha .
$$

\section{The Classification of SPACE-Like surfaces in $\mathbf{Q}_{1}^{n}$ With $\Phi=0$}

Let $x: \mathbf{M} \rightarrow \mathbf{Q}_{1}^{n}$ be a space-like surface in $\mathbf{Q}_{1}^{n}$ with vanishing conformal form, i.e., $\phi_{\alpha}=0,3 \leq \alpha \leq n$. Then the fundamental equations come into

$$
\begin{gathered}
\psi_{\bar{z}}=\frac{1}{2} e^{2 \omega} K_{z}, \quad \bar{\psi} \Omega_{\alpha}=\psi \bar{\Omega}_{\alpha}, \quad e^{-4 \omega} \sum_{\alpha} \Omega^{\alpha} \Omega_{\alpha}=-\frac{1}{4}, \\
\left(\Omega_{\alpha}\right)_{\bar{z}}=-\sum_{\beta} \Omega^{\beta} \bar{A}_{\beta \alpha}, \quad A_{\alpha \beta}=-A_{\beta \alpha} .
\end{gathered}
$$

It follows from (3.2) that

$$
\left(\sum_{\alpha} \Omega^{\alpha} \Omega_{\alpha}\right)_{\bar{z}}=0
$$

thus the globally defined 4-form $\sum_{\alpha} \Omega^{\alpha} \Omega_{\alpha} d z^{4}$ is holomorphic on M. From (3.1) we get

$$
\bar{\psi} \sum_{\alpha} \Omega^{\alpha} \Omega_{\alpha}=\sum_{\alpha} \Omega^{\alpha} \bar{\Omega}_{\alpha}=-\frac{1}{4} e^{4 \omega} \psi
$$

Immediately we have the following lemma.

Lemma 3.1. Let $x: \mathbf{M} \rightarrow \mathbf{Q}_{1}^{n}$ be a surface in $\mathbf{Q}_{1}^{n}$ with vanishing conformal form. Then the conformal invariant $\Psi=\psi d z^{2}$ is holomorphic on $\mathbf{M}$. Then $\Psi$ vanishes identically or the zero points of $\Psi$ are isolated.

First we consider the case that $\Psi \equiv 0$. Thus from (3.1), $K$ must be a constant. Then we get from (2.3) that

$$
N=\frac{1}{8}(4 K-1) Y+\mathbf{c}
$$

for some constant vector $\mathbf{c} \neq 0$ in $\mathbf{R}_{2}^{n+2}$. Therefore $x$ is a conformal isotropic surface, i.e., $x$ is a stationary surface with constant curvature in $\mathbf{R}_{1}^{n}, \mathbf{S}_{1}^{n}$, or $\mathbf{H}_{1}^{n}$ (see [7]). 
Now we come to discuss the case that the zero points of $\Psi$ are isolated. In this case we can cut $\mathbf{M}$ by some disjoint curves $C_{i}$ to get a simply connected domain $\mathbf{U}=\mathbf{M} \backslash \sum_{i} C_{i}$ such that $x: \mathbf{U} \rightarrow \mathbf{Q}_{1}^{n}$ is a surface with $\Psi \neq 0$ on $U$. By choosing a complex coordinate if necessary, we may assume that $\psi \equiv 1$ on $\mathbf{U}$. It follows from (2.1) and (3.1) that $K=-4 e^{-2 \omega} \omega_{z \bar{z}}=0$. By (3.3) we know that $\left\{\Omega_{\alpha}\right\}$ are real functions. We define a global real vector field $E \in \mathbf{V}$ by

$$
E=2 e^{-2 \omega} \sum_{a} \Omega^{\alpha} E_{\alpha},
$$

then by (3.2) we have $\langle E, E\rangle=-1$. Choosing $\tilde{E}_{3}=E$ and expanding it to a local orthonormal basis of the conformal normal bundle $\left\{\tilde{E}_{3}, \tilde{E}_{4}, \cdots, \tilde{E}_{n}\right\}$, one can easily verify that

$$
\tilde{\Omega}_{3}=-\frac{1}{2} e^{2 \omega}, \tilde{\Omega}_{4}=\cdots=\tilde{\Omega}_{n}=0
$$

Using (3.3), we get

$$
\begin{gathered}
\left(\tilde{\Omega}_{3}\right)_{\bar{z}}=-\sum_{\beta=3}^{n} \tilde{\Omega}^{\beta} \bar{A}_{\beta 3}=-\tilde{\Omega}_{3} \bar{A}_{33}=0, \\
0=\left(\tilde{\Omega}_{\alpha}\right)_{\bar{z}}=-\sum_{\beta \neq \alpha} \tilde{\Omega}^{\beta} \bar{A}_{\beta \alpha}=-\tilde{\Omega}_{3} \bar{A}_{3 \alpha}, \alpha>3 .
\end{gathered}
$$

Thus $\omega$ is a constant and $A_{3 \alpha}=0, \forall \alpha$. Now the structure equations read

$$
\begin{aligned}
N_{z} & =\frac{1}{8} Y_{z}+e^{-2 \omega} Y_{\bar{z}}, \\
Y_{z z} & =-\frac{1}{2} Y-\frac{1}{4} e^{2 \omega} \tilde{E}_{3}, \quad Y_{z \bar{z}}=\frac{1}{16} e^{2 \omega} Y-\frac{1}{2} e^{2 \omega} N, \\
\left(\tilde{E}_{3}\right)_{z} & =\frac{1}{2} Y_{\bar{z}}, \quad\left(\tilde{E}_{\alpha}\right)_{z}=\sum_{\beta} A_{\alpha}^{\beta} \tilde{E}_{\beta}, \quad \alpha>3 .
\end{aligned}
$$

A surface is said to be full in $\mathbf{Q}_{1}^{n}$ if $x(\mathbf{M})$ does not lie in any totally umbilic $\mathbf{Q}^{n-1}$ of $\mathbf{Q}_{1}^{n}$. We assume that $n \geq 4$. Then fixing a point $p \in U$, we can find a constant vector $\xi \in \mathbf{R}_{2}^{n+2}$ with $\langle\xi, \xi\rangle=1$ such that

$$
\begin{aligned}
& \langle Y(p), \xi\rangle=0, \quad\langle N(p), \xi\rangle=0, \quad\left\langle Y_{u}(p), \xi\right\rangle=0, \\
& \left\langle Y_{v}(p), \xi\right\rangle=0, \quad\left\langle\tilde{E}_{3}(p), \xi\right\rangle=0 .
\end{aligned}
$$

We define real functions

$$
\begin{array}{r}
f_{1}=\langle Y, \xi\rangle, \quad f_{2}=\langle N, \xi\rangle, \quad f_{3}=\left\langle Y_{u}, \xi\right\rangle, \\
f_{4}=\left\langle Y_{v}, \xi\right\rangle, f_{5}=\left\langle\tilde{E}_{3}, \xi\right\rangle .
\end{array}
$$

Then by (3.11)-(3.13) we can find constants $\left\{a_{\lambda \mu}\right\}$ and $\left\{b_{\lambda \mu}\right\}$ such that

$$
\left(f_{\lambda}\right)_{u}=\sum_{\mu} a_{\lambda \mu} f_{\mu}, \quad\left(f_{\lambda}\right)_{v}=\sum_{\mu} b_{\lambda \mu} f_{\mu}, \quad 1 \leq \lambda, \mu \leq 5 .
$$

By (3.12) and the uniqueness of the linear PDE (3.14) we get $f_{\lambda} \equiv 0$. In particular, $f_{1}=\langle Y, \xi\rangle=0$ on $U$, which implies that $\langle Y, \xi\rangle=0$ on $\mathbf{M}$. If $x$ is full, then $n$ must be 3 . 


\section{ACKNowledgements}

The authors would like to express their gratitude to Professors Changping Wang and Haizhong Li for their warm-hearted instruction and inspiration.

\section{REFERENCES}

[1] Wang, C.P., Moebius geometry of submanifolds in $S^{n}$, Manuscripta Math. 96 (1998), 517534. MR.1639852(2000a:53019)

[2] Li, H.Z., Wang, C.P., Surfaces with vanishing Moebius form in $\mathbb{S}^{n}$, Acta Math. Sinica, Engl. Ser. 19 (2003), 671-678. MR2023361 (2004j:53076)

[3] Bryant, R.L., Minimal surfaces of constant curvature in $\mathbb{S}^{n}$, Trans. Amer. Math. Soc. 290 (1985), 259-271. MR787964 (87c:53110)

[4] Deng, Y.J., Wang, C.P., Willmore surfaces in Lorentzian space, Sci. China Ser. A 35 (2005), $1361-1372$

[5] Nie, C.X., Ma, X., Wang, C.P., Conformal CMC-surfaces in Lorentzian space forms, Chin. Ann. Math., Ser. B, 28 (2007), 299-310. MR2339435 (2008e:53022)

[6] Alias, L.J., Palmer, B., Conformal geometry of surfaces in Lorentzian space forms, Geometriae Dedicata 60 (1996), 301-315. MR1384435 (97f:53099)

[7] Nie, C.X., Li, T.Z., He, Y.J., Wu, C.X., Conformal isoparametric hypersurfaces with two distinct conformal principal curvatures in conformal space, Sci. China Ser. A 53 (2010), 953-965. MR2640180 (2011c:53133)

[8] Blaschke, W., Vorlesungen über Differentialgeometrie, Vol. 3, Springer, Berlin, 1929.

[9] Hertrich-Jeromin, U., Introduction to Möbius Differential Geometry, London Math. Soc. Lecture Note Series, Vol. 300, Cambridge University Press, Cambridge, 2003. MR2004958 (2004g:53001)

[10] O'Neill, B., Semi-Riemannian Geometry with Applications to Relativity, Pure and Applied Mathematics, 103, Academic Press, New York, 1983. MR719023 (85f:53002)

Faculty of Mathematics and Computer Sciences, Hubei University, Wuhan 430062 , People's Republic of China

E-mail address: chxnie@163.com 